\title{
Desafíos transdisciplinares en la universidad: Leln Lenguaje Inclusivo para fomentar el aprendizaje del alumnado
}

\section{Interdisciplinary challenges at University: Leln Inclusive Language to promote students' learning}

juan Pablo Mora GutiérRez

ORCID: https://orcid.org/0000-0002-8614-5117

Universidad de Sevilla

Departamento de Lengua Española, Lingüística y Teoría de la Literatura

jmora@us.es

DOI: http://dx.doi.org/10.12795/9788447231003.076

Pp.: 1623-1653 


\section{Contexto del ciclo de mejora en el aula}

Llevo desde el curso 2015-2016 impartiendo la asignatura de tercero del grado en Filología Hispánica Lingüística Aplicada LA con metodologías activas como Flipped Learning, Aprendizaje Basado en Proyectos o Aprendizaje Basado en Retos. En los tres cursos pasados (2017-2018, 2018-2019 y 2019-2020), en el marco de un proyecto de innovación docente de la US denominado El Reto en la Educación Superior: Aprendizaje con ECO, he usado el modelo docente de Aprendizaje Basado en Retos con metodología de Pensamiento de Diseño (Design Thinking en inglés, DT). En los dos últimos cursos la metodología DT ha sido adaptada a una metodología que hemos denominado ECO, reduciendo las fases del DT a tres, Explorar, Crear y Ofrecer.

\begin{tabular}{|c|c|c|}
\hline \multicolumn{2}{|c|}{ ETAPAS } & \multirow[t]{2}{*}{ PASOS } \\
\hline ECO & DT & \\
\hline \multirow{2}{*}{ EXPLORAR } & EMPATIZA & $\begin{array}{l}\text { Observar } \\
\text { Comprender } \\
\text { Descubrir }\end{array}$ \\
\hline & DEFINE & $\begin{array}{l}\text { Procesar } \\
\text { Elegir un punto de vista } \\
\text { Formular el reto }\end{array}$ \\
\hline \multirow{3}{*}{ CREAR } & IDEA & $\begin{array}{l}\text { Imaginar } \\
\text { Establecer criterios de selección } \\
\text { Elegir entre alternativas } \\
\text { Decidir idea/solución }\end{array}$ \\
\hline & PROTOTIPA & $\begin{array}{l}\text { Construir } \\
\text { Someter a interacción } \\
\text { Reconstruir }\end{array}$ \\
\hline & EVALÚA & $\begin{array}{l}\text { Ganar comprensión } \\
\text { Aplicar retroalimentación } \\
\text { Refinar solución }\end{array}$ \\
\hline OFRECER & COMPARTE & $\begin{array}{l}\text { Implementar plan de acción } \\
\text { Difundir } \\
\text { Evaluar } \\
\text { Contagiar } \\
\text { Celebrar } \\
\end{array}$ \\
\hline
\end{tabular}

Ciclos de Mejora en el Aula (2020). Experiencias de Innovación Docente de la US Esta obra se distribuye con la licencia Creative Commons 
Tabla 1: Equivalencia de las fases de DT con la metodología ECO (adaptación del DT a la US que se ha llevado a cabo en el proyecto de innovación docente mencionado)

Esta metodología es enteramente constructivista, con el alumnado creando desde cero retos o proyectos que tiene que identificar, definir e implementar en el transcurso del cuatrimestre. Por lo tanto el foco de la asignatura no es la transmisión de contenidos ni la enseñanza centrada en el docente, sino que prácticamente todo el conocimiento que se genera es producido por el alumnado trabajando en equipos. Diría que el foco no está ni siquiera ni por importante que sea en el aprendizaje del alumnado, como se suele defender en otros modelos de innovación docente, sino que en lo que se centra la docencia DT/ECO es en la sociedad, sus necesidades y los desafíos que se le plantean a las personas en un mundo tan complejo y en crisis como el actual. El alumnado tiene que identificar y desarrollar retos y desafíos que afecten a personas en los que pueda aplicar tanto los conocimientos que tiene como lingüistas en formación así como los que necesitará adquirir en la asignatura en cuestión para poder dar una solución a esa necesidad identificada y reto elegido. De esta forma se aprende de una manera experiencial, interactuando con personas y con expertos y aplicando los conocimientos que ya se tienen e integrando los nuevos que son el objetivo de la asignatura para poder abordar el reto definido, aportar una solución original y creativa y poder ofrecer los resultados a la sociedad. Los excelentes resultados que se obtienen y las competencias transversales que se ejercitan se pueden ver claramente en los dos padlets de LA de los cursos 2019-2020 y 2018-2019, a través de este enlace: https://padlet.com/jmora4

En el curso 2019-2020 se matricularon en el grupo de mañana de LA 102 estudiantes, 40 más que en cursos anteriores. Se establecieron 18 equipos de entre 3 y 8 personas. 
Participamos en la Noche Europea de los Investigadores en septiembre de 2019 y organizamos dos Zocos de Lenguas y Culturas, uno en la Facultad de Filología y el segundo en la residencia Flora Tristán situada en el Polígono Sur. Además, los mejores retos se presentaron en el V ECOshowUS del proyecto de innovación docente en enero de 2020. En la columna de hitos del padlet de la asignatura se puede acceder a todas las grabaciones y evidencias de nuestra participación en esos eventos.

La única directriz que se dio para el establecimiento de los equipos fue que el alumnado se agrupara por el interés común en una temática determinada con el único requisito de que, si resultaba posible, en cada equipo hubiera alumnado español y alumnado Erasmus. El alumnado fue calificado a través de un portafolios grupal en el que se recogía lo más sobresaliente del proyecto desarrollado y un diario reflexivo individual en el que se tenía que reflexionar sobre todo aquello que hubiera dejado huella y sobre lo que se hubiera aprendido. En ese diario individual se tenía que incluir un apartado de evaluación con una autoevaluación, una evaluación de los demás miembros del equipo, de los demás proyectos de la clase y de la metodología y del docente.

Se intentó que el alumnado se olvidara de esta evaluación sumativa por medio de una evaluación continua formativa en la que se llevaron a cabo varias presentaciones en clase y en los eventos en los que participamos. Además, se observó y dio feedback al alumnado en todo momento en el aula mientras trabajaba en sus proyectos, se llevó a cabo evaluación por pares y se permitió al alumnado organizar debates o clases grupales sobre los temas de sus retos, proponer y escribir ensayos sobre los demás retos y participar en diversas actividades enriquecedoras como asistencia a jornadas, cursos o eventos relacionados con la lingüística aplicada, participar como voluntarios en 
la organización del Mercadillo de Babel (actividad de enseñanza de idiomas creada por el profesor Christoph Ehlers para su alumnado del máster MASELE) o actividades de intercambio tándem de idiomas con alumnado extranjero.

El curso pasado el confinamiento no afectó a esta asignatura al ser de primer cuatrimestre, pero sí que lo hizo con las otras dos asignaturas de grado que tenía asignadas en el segundo cuatrimestre, Temas de Lingüística de cuarto y Lingüística de primero de Filología Hispánica. En ambas asignaturas también vengo usando en los últimos cursos el modelo de Aprendizaje Basado en Retos. La adaptación a la situación de confinamiento fue bastante complicada pues dificultó una de las fases clave de la metodología DT/ECO, que es la de implementar y ofrecer a la sociedad la solución creada para la necesidad o reto detectado. También tuvimos que cancelar la participación del alumnado y sus proyectos en los eventos que teníamos programados, el IV Zoco de Lenguas y Culturas que estábamos organizando nosotros y nuestro alumnado junto con las personas solicitantes de protección internacional a las que dábamos clase de ELE en la facultad. Este Zoco estaba previsto para el 2 de abril de 2020. Íbamos a llevar los retos del alumnado también al Salón del Estudiante de la US en abril, a la quinta edición de la Muestra Anual de Comunicación MUAC prevista para el 24 de marzo, al Encuentro Anual de Blogs Educativos EABE de Sanlúcar de Barrameda previsto también por esas fechas. Solo se llevó a cabo en junio, pero de forma no presencial, el VI ECOshowUS del proyecto de innovación docente, El Reto en la Educación Superior: Aprendizaje con ECO. Este evento fue organizado y presentado por el propio alumnado. Aprovechamos también para participar con gran éxito en la Feria de la Ciencia que se tuvo que celebrar de forma virtual en junio de 2020.

A pesar de las dificultades y del reto que supuso la pandemia y la docencia online, quedó patente durante el 
confinamiento que el modelo docente de ABR con DT/ECO fue mucho más efectivo y resiliente que los modelos centrados en la enseñanza y en la transmisión del conocimiento por parte del profesorado. El alumnado adaptó sus proyectos a las circunstancias y trabajó más que nunca logrando un nivel de creatividad y de profundidad en casi todos los equipos raramente alcanzado antes. En el padlet que recoge lo creado por el alumnado y los hitos de la asignatura de Temas de Lingüística General 2019-2020 se puede comprobar la veracidad de esta afirmación. Basta con compararlo con los padlets de cursos anteriores. Una circunstancia que ayudó al éxito conseguido fue que en las semanas de clases presenciales antes del confinamiento se había logrado establecer una relación de confianza con el alumnado. Además, los proyectos estaban ya encaminados con la fase EXPLORA, que concluye con la definición del reto, casi finalizada. El CIMA de asignatura completa que se describe en esta ocasión se ha llevado a cabo en los dos grupos de la asignatura Lingüística Aplicada del Grado en Filología Hispánica. Es una asignatura optativa de tercero. Este curso se han matriculado 80 estudiantes entre ambos grupos, bastantes menos que en cursos anteriores, debido a la menor presencia de alumnado Erasmus debida a la pandemia. Además, han cursado la asignatura tres estudiantes del doble grado en Filología Clásica y en Filología Hispánica. El primer mes de clase la docencia se llevó a cabo de una forma semipresencial con un tercio del alumnado en el aula y dos tercios siguiendo las clases desde sus casas. A partir de la segunda semana de noviembre toda la docencia pasó a ser online.

\section{Diseño previo del CIMA}

La innovación docente que he llevado a cabo este cuatrimestre se centra en la cooperación con colegas y alumnado de otras facultades y universidades y en la no obligación por parte del alumnado de usar ninguna metodología 
impuesta para llevar a cabo los retos en equipo. En su lugar se ha proporcionado información sobre diferentes metodologías de aprendizaje activo que se adaptan bien al Aprendizaje Basado en Retos como DT/ECO, Aprendizaje Servicio ApS, Investigación-Acción-Participación e incluso se permitió que se llevaran a cabo proyectos de investigación más tradicionales sin necesidad de implementar lo averiguado, ya que la situación de pandemia lo dificultaba. El tener que decidir entre las diferentes metodologías la que mejor se adaptara a su reto o proyecto se convirtió así en una oportunidad más de aprendizaje para el alumnado. Por otro lado, ha sido extraordinariamente enriquecedor tener que cooperar con alumnado de otras facultades y universidades. Con esa cooperación se intentó lograr suplir la falta de heterogeneidad en los equipos causada por el menor número de Erasmus matriculados en comparación con cursos anteriores.

El estudiantado de LA y yo hemos cooperado con Vicente Manzano Arrondo, de la Facultad de Psicología de la US, y su alumnado de las asignaturas Psicometría de tercero y Metodología Cualitativa, de cuarto, con Concha Torres de la Facultad de Educación y miembro también de la REFID y el alumnado de Lengua española y su didáctica de tercero del grado en Educación Primaria, con Inés Lucas, también de la Facultad de Educación y su alumnado de la asignatura de segundo del grado en Educación Primaria Desarrollo de habilidades lingüísticas y su aplicación didáctica en educación primaria y con Concha Báez, de la Universidad de Vigo, y su alumnado de primero del grado en Traducción e Interpretación de la asignatura Lengua española oral y escrita. También se ha sumado alumnado de la profesora María del Rosario Martínez Navarro de la asignatura Técnicas de Investigación literaria de cuarto de Filología Hispánica. Además, colaboraba en esta iniciativa la profesora Victoria Camacho, que tenía asignados algo más de dos créditos del grupo de tarde de LA, aunque por una baja médica tuvo que dejar esa docencia cuando solo llevaba una semana impartida.

Ciclos de Mejora en el Aula (2020). Experiencias de Innovación Docente de la US Esta obra se distribuye con la licencia Creative Commons 
Para facilitar el trabajo de los equipos transdisciplinarios establecimos una serie de retos marco en los que el alumnado se apuntó de acuerdo con sus inquietudes e intereses y empezó a trabajar en diversos equipos formados preferiblemente por entre 4 y 6 alumnos de las diferentes asignaturas. La descripción de algunos de estos retos marco se puede consultar en el Anexo. Cada docente se encargó de supervisar a su propio alumnado. No obstante, se acordó que una persona del equipo de docentes se preocuparía de reunirse periódicamente y de supervisar el trabajo de los diversos equipos transdisciplinarios de un mismo reto o desafío marco. Cada equipo diseñó su propio proyecto o reto dentro del marco general decidiendo para qué colectivo trabajaría y en qué consistiría exactamente su proyecto o reto. Yo me encargué de supervisar y asesorar el trabajo del desafío marco Leln sobre Lenguaje Inclusivo. Cinco de los 37 equipos transdisciplinares que se establecieron se ocuparon de ese tema. Tres de ellos contaban con alumnado de Lingüística Aplicada LA. El desafío marco más popular fue Discursos de Odio, propuesto y guiado por Concha Torres, de la Facultad de Educación, con 9 equipos.

Aparte de los grandes temas marco propuestos por el profesorado, se incentivó que el alumnado de LA propusiera otros retos siempre que tuvieran que ver con la lingüística aplicada y con el futuro profesional de los egresados del grado en Filología Hispánica. Se hizo lo posible por intentar que en esos proyectos también hubiera alumnado de otras facultades. Finalmente, de los 28 equipos formados con alumnado de LA solo uno de ellos no logró atraer a alumnado de otras facultades. No obstante, contó con una alumna de intercambio de China y dos españoles, lo que dotó a ese reto de suficiente diversidad.

Ciclos de Mejora en el Aula (2020). Experiencias de Innovación Docente de la US Esta obra se distribuye con la licencia Creative Commons 
En cursos pasados ya había intentado que mi alumnado colaborara en sus proyectos/retos con alumnado de otras facultades en el marco del proyecto de innovación docente DT/ECO. Solo lo conseguí en el caso del proyecto "Los Pajaritos Hablan" en el curso 2018-2019. Este equipo colaboró con un equipo de la asignatura impartida por la profesora Noelia Melero Educación Comunitaria y de las Personas Adultas del Grado en Pedagogía. Sin embargo, la experiencia no fue del todo satisfactoria pues surgieron los problemas habituales del trabajo en equipo vinculados a la comunicación dentro del equipo, la carga de trabajo de cada miembro y lo que se percibió como una deficiente colaboración por parte del alumnado de ambos grados. Tras la experiencia vivida durante el confinamiento y lo aprendido por el estudiantado sobre el trabajo en equipo online el curso pasado, en esta ocasión se ha conseguido que el alumnado trabaje eficientemente en los equipos transdisciplinarios de forma virtual. También ha sido un factor la experiencia que he ido acumulando en los últimos años en la gestión del trabajo en equipo y en la prevención de las dificultades y problemas que conlleva esa forma de trabajo. Para prevenir esas dificultades suelo invitar a clase a alumnado que había experimentado problemas en cursos pasados y también a alumnado al que le había ido bien en el trabajo en equipo. Suelen hablar de lo conseguido en sus retos para que el alumnado no familiarizado con $A B R$ vea lo que se puede conseguir con este modelo docente. Al mismo tiempo advierten gráficamente de los problemas y dificultades con las que se va a encontrar el nuevo alumnado en sus trabajos en equipo. No obstante, por mucho que se señalan los posibles problemas, solo se aprende a superarlos cuando se experimentan y viven en las propias carnes en cada equipo.

Ciclos de Mejora en el Aula (2020). Experiencias de Innovación Docente de la US Esta obra se distribuye con la licencia Creative Commons 


\section{Aplicación del CIMA}

En las clases de la primera semana de octubre, que coincidió con la primera semana del cuatrimestre, se dieron algunas nociones básicas y fundamentales de cada uno de los temas que componen el programa de la asignatura. Un resumen gráfico de los principales contenidos de la materia se puede ver en este mapa conceptual, creado por Raquel Benítez Burraco, profesora del grupo de tarde junto con Juan Pablo Mora y Victoria Camacho. Raquel Benítez Burraco solo tenía asignada docencia para una sesión de clase que impartió online el 5 de octubre, la primera clase del curso. Esta clase de 80 minutos consistió en una breve introducción a las principales ramas de la LA (Véase Figura 1)

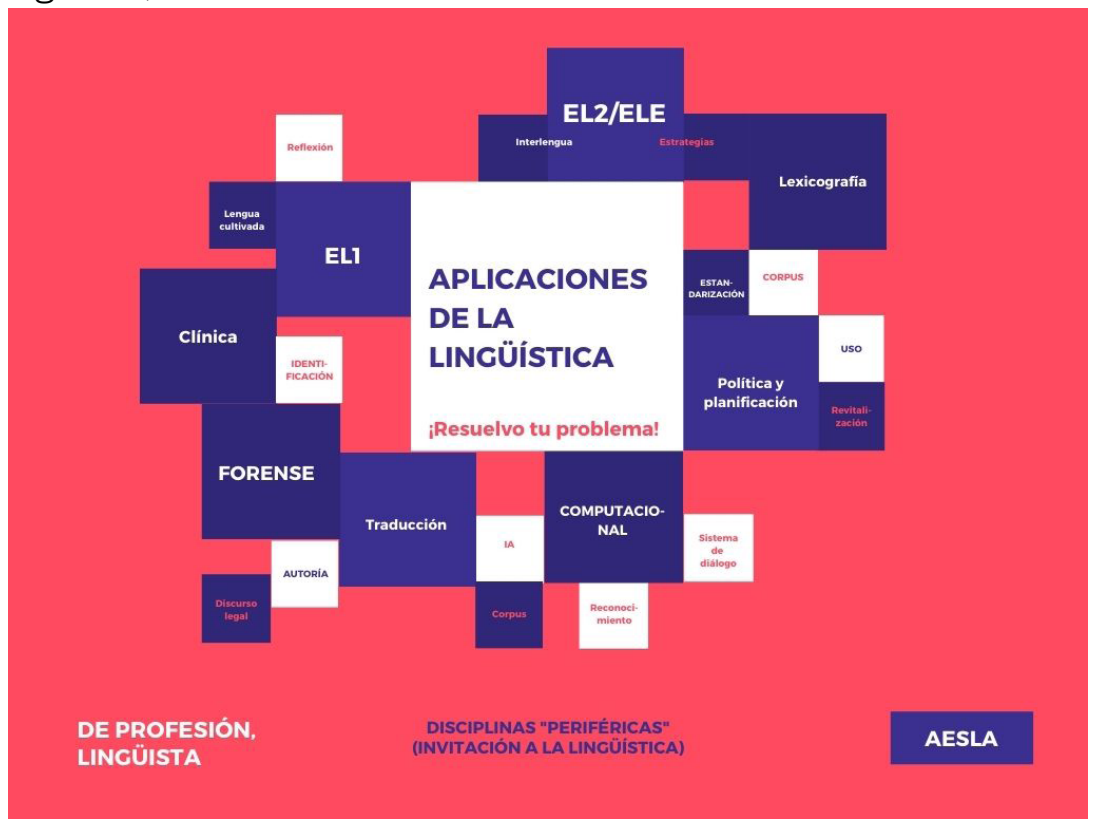

Figura 1: Mapa conceptual con las principales ramas de la Lingüística Aplicada (Raquel BB)

El vídeo de la grabación de esa primera clase así como la lectura introductoria en la que estaba basada se subieron a la aplicación PERUSALL de lectura y visionado Ciclos de Mejora en el Aula (2020). Experiencias de Innovación Docente de la US Reconocimiento-NoComercial-SinObraDerivada Internacional (CC BY-NC-ND 4.0.) 
de vídeos de forma colaborativa. Se mandó al alumnado como tarea escribir comentarios y preguntas en Perusall tanto al vídeo como a la lectura. Se estableció así un diálogo que fue muy enriquecedor y que fue complementado con una tarea en la plataforma que consistía en entregar un resumen de lo aprendido en el tema de introducción a través de un mapa conceptual, un podcast, un vídeo, un esquema o una representación visual de ese conocimiento adquirido.

No obstante, dado que el temario es mucho más amplio de lo que se puede abarcar en profundidad en una asignatura cuatrimestral de 6 créditos, el foco lo suelo poner en aquellos temas que interesen de forma especial al alumnado según sus inclinaciones y vocación profesional. De esta forma, el conocimiento de los diferentes temas del programa de la materia se suele construir fundamentalmente en los diversos retos que se definen y en los proyectos que el alumnado lleva a cabo. Antes incluso de empezar las clases se suele pedir al estudiantado que rellene un cuestionario inicial para recabar información de su vocación, sus intereses, de las redes sociales que usa y de la experiencia previa que hubiera podido tener con metodologías de aprendizaje activo en cursos anteriores. En esta ocasión, este cuestionario lo habían respondido 60 estudiantes antes del primer día oficial de clase a primeros de octubre de 2020.

Como se ha comentado anteriormente, lo esencial y original de este CIMA, en comparación con los llevados a cabo en cursos anteriores, ha sido que el alumnado, agrupado en equipos heterogéneos--españoles y extranjeros y alumnado de otras facultades y universidades-ha llevado a cabo un proyecto o reto transdisciplinario que ha dado respuesta a alguna necesidad o problema de la sociedad en los que la lingüística pueda llegar a jugar un papel clave y relevante. De esta forma se ha intentado emular mejor 
la realidad que el alumnado se encontrará en el mercado de trabajo y en las empresas en las que prevalece el trabajo en equipos interdisciplinares, única manera de abordar cualquier reto complejo con garantías de éxito.

Teniendo en cuenta que el año 2019 fue el año internacional de las lenguas indígenas, autóctonas u originarias y que la ONU ha aprobado una década internacional a partir de 2022 para actuar sobre los problemas y retos a los que se enfrentan estas mismas lenguas y sus culturas asociadas en el marco de los Objetivos para un Desarrollo Sostenible (ODS), muchos de los retos que se han diseñado han versado sobre la discriminación e incluso la opresión lingüística que muchas personas sufren a causa de la lengua o de la variedad lingüística que hablan, o por tener algún trastorno del lenguaje, o por pertenecer a un grupo minoritario o minorizado. Los temas de la Lingüística Aplicada que se han tratado a través de estos proyectos o retos han sido los de Actitudes Lingüísticas o Percepción Lingüística, Política Lingüística, Documentación y Revitalización de Lenguas, Lenguaje y Poder, Lingüística Clínica y temas relacionados con el bilingüismo, el contacto de lenguas, la traducción, la educación y la enseñanza de idiomas. La Lingüística Forense, la Lingüística Computacional y el Procesamiento del Lenguaje Natural fueron elegidos también como ámbitos de estudio por parte de varios de los equipos transdisciplinares que se formaron y que prefirieron elegir temas ajenos a los retos marco propuestos por los docentes.

La fase inicial de exploración del reto se desarrolló desde el 13 de octubre hasta el 30 de noviembre. Lo conseguido en estas semanas fue llevado a la Noche Europea de los Investigadores el 27 de noviembre de 2020, por medio de esta actividad en la que el alumnado de LA fue protagonista:

Ciclos de Mejora en el Aula (2020). Experiencias de Innovación Docente de la US Esta obra se distribuye con la licencia Creative Commons 
https://lanochedelosinvestigadores.fundaciondescubre.es/actividades/investigamos-sobre-diversidad-linguistica-y-cultura-el-reto-de-la-ciencia-ciudadana/

En esa actividad online, por la que recibimos la felicitación de la organización de la Noche por ser la más concurrida de las que tuvieron lugar en la sede de Sevilla, el alumnado llevó a cabo una simulación de un programa de radio Onda 25 en el que se presentaron como futuros profesionales de diversos ámbitos de la lingüística y contaron de una forma muy creativa e informativa en qué consisten algunos de los retos en los que se habían embarcado: https://youtu.be/Lu_RmtBDSBI

Previamente, en cuatro sesiones de clase de esa misma semana final de noviembre, se llevaron a cabo las presentaciones de la fase Explora de los 27 proyectos interdisciplinares que se han llevado a cabo y que han cubierto la inmensa mayoría de las ramas de la lingüística aplicada. Para suplir la menor atención a algunos temas fundamentales como la enseñanza de la L1 y de las segundas lenguas, la biolingüística o la lingüística computacional, se invitó a especialistas en cada una de esas áreas para que impartieran clases introductorias a dichos ámbitos de la LA.

Como se ha comentado anteriormente, la otra gran aportación de este CIMA ha sido que el alumnado de cada equipo ha tenido que decidir el enfoque o metodología que mejor se adaptara a sus retos. La inmensa mayoría de los equipos, siguiendo el ejemplo de cursos anteriores, optaron por llevar a cabo un proyecto DT/ECO. También se optó por ApS (Aprendizaje Servicio), e incluso Investigación-Acción-Participación, aunque en bastante menor medida.

El último día de clase de cada semana de ambos grupos se dedicaron a un debate o a una actividad especial

Ciclos de Mejora en el Aula (2020). Experiencias de Innovación Docente de la US Esta obra se distribuye con la licencia Creative Commons 
propuesta por algún equipo. Se han llevado a cabo debates sobre lenguaje inclusivo, lenguas minoritarias, Inteligencia Artificial y Lingüística Computacional, bilingüismo, Lingüística Clínica, en concreto disfemia y sobre enseñanza a alumnado con Asperger, Lingüística Forense, Discriminación del andaluz y dentro de este del ceceo, sobre los discursos del odio y en concreto sobre la islamofobia, sobre comunicación con personas sordociegas y un debate final sobre política lingüistica en España y en concreto sobre las lenguas vehiculares en la educación a raíz de la polémica suscitada por la eliminación del texto de la ley Celáa o LOMLOU de la mención explícita al castellano como lengua vehicular en la educación. Algunos temas, como el del lenguaje inclusivo y la lengua vehicular en la educación son muy controvertidos y desgraciadamente están siempre de actualidad en nuestra sociedad, con lo que resultan de mucho interés para el alumnado, que aprende mucho a través del debate y la discusión en clase de estos temas polémicos y controvertidos.

Se había recomendado en el proyecto docente de la asignatura que cada alumna y cada alumno dedicara a los proyectos o retos en equipo al menos dos tercios de las 150 horas que corresponden a los seis créditos de la asignatura. Las 50 horas restantes se deberían dedicar a la lectura de la bibliografía básica recomendada, a escribir al menos un ensayo opcional entre los propuestos por el docente y por los demás equipos de la clase, a participar en cursos, conferencias, congresos y otras actividades, como puede ser un intercambio lingüístico Tándem, el mercadillo de Babel del alumnado del máster MASELE, algún MOOC, proyectos de recogida de datos a través de las redes sociales, debates en clase, retos puntuales, etc. Todos los equipos que participaron en proyectos transdisciplinarios tuvieron que presentar además sus proyectos en un congreso que organizó el alumnado de psicología de Vicente Manzano. Ese congreso final tuvo lugar el sábado

Ciclos de Mejora en el Aula (2020). Experiencias de Innovación Docente de la US Esta obra se distribuye con la licencia Creative Commons 
23 de enero de 2021. Ese día y durante 6 horas se debatió sobre el conocimiento construido por el alumnado en los 37 proyectos transdisciplinares que se habían llevado a cabo. Previamente cada asistente a una de las cuatro sesiones del congreso tenía que haber visto los vídeo-resumen de 5 minutos que cada equipo había tenido que crear con la antelación suficiente. Se pueden ver todos esos vídeos así como la grabación de las seis horas de debate en la web del congreso, creada como todo lo demás por el equipo organizador del evento: https://kongres02021.wordpress.com/

Los proyectos/retos que así lo quisieron pudieron participar también en el VII ECOshowUS del proyecto de innovación docente ECO que se celebró hace dos días, el viernes 29 de enero de 2021: https://www.us.es/actualidad-de-la-us/ mas-de-500-estudiantes-participan-en-la-septima-edicion-de-ecoshowus. En ese evento se presentaron casi 50 retos de proyectos de alumnado de las distintas facultades y ramas de conocimiento que participan en el proyecto de innovación docente, además de diversas obras de artes creadas por el alumnado de Bellas Artes del profesor Ramón Blanco Barrera, con una exposición presencial en el Panteón de los Sevillanos Ilustres. El alumnado de LA participó con un podcast que crearon dos de las alumnas de la asignatura con experiencia gracias al programa Radio Areúsa de Radius. En este podcast, que se situó en el año 2041, conmemorando los 20 años de la participación en la Noche Europea de los Investigadores de 2021, el alumnado que quiso participar se presentó como profesionales de la lingüística cuya carrera se había lanzado gracias a los proyectos que habían iniciado en el marco de LA en el curso 2020-2021. Esta original forma de contar lo hecho en esos proyectos durante el cuatrimestre se puede ver en esta grabación de YouTube https://youtu.be/te3krz5M730. Además se presentaron tres de los proyectos de la asignatura que habían destacado en el Congreso del 23 de enero,

Ciclos de Mejora en el Aula (2020). Experiencias de Innovación Docente de la US Esta obra se distribuye con la licencia Creative Commons 
Hablando con Hans, sobre alumnado con Asperger, L1 y las Emociones, y La Lengua de la Lluvia, de revitalización de la lengua tu'un savi o lengua mixteca. Asimismo, presentaron tres equipos de las asignaturas de literatura de María del Rosario Martínez Navarro.

\section{Evaluación de lo aprendido}

En el proyecto docente de la asignatura se había establecido lo siguiente en cuanto a la evaluación:

El alumnado tendrá que elegir entre una evaluación continua o una evaluación a través de un único examen final que será oral. El sistema de evaluación recomendado a todo el alumnado es la evaluación continua. Para determinar la nota final será necesario llevar a cabo las siguientes tareas y actividades:

1) Entrega de un portafolios final grupal del proyecto realizado en equipo. El portafolios de la fase explora se entregará a primeros de diciembre. Cada equipo tendrá que llevar a cabo dos presentaciones en clase de cada proyecto, una intermedia y otra final. Asimismo, se creará también un póster o cartel y un vídeo de menos de cinco minutos en los que se resuma en otro medio lo realizado en por lo menos uno de los dos proyectos/retos del equipo. Este trabajo grupal equivaldrá al $50 \%$ de la nota.

2) Entrega de un diario reflexivo de la asignatura enriquecido con un apartado de evaluación que incluirá obligatoriamente una autoevaluación, una evaluación de pares y una evaluación del docente y la asignatura. Este diario de clase tendrá tres momentos de entrega, uno inicial en el que se escribirá una autobiografía lingüística y que se entregará el viernes 16 de octubre, uno intermedio que se entregará la primera semana de diciembre tras la participación en la Noche de los Investigadores y una entrega 
final el 1 de febrero. El diario reflexivo contará el 30\% de la calificación final.

3) Realización durante el mes de diciembre de una actividad obligatoria de autoevaluación y evaluación de pares en la plataforma de enseñanza virtual que valdrá el $10 \%$ de la calificación final.

4) Participación en clase y realización de otras actividades opcionales como ensayos, asistencia a conferencias, congresos y MOOC relacionados con la lingüística, intercambio lingüístico, proyectos de recogida y difusión a través de las redes sociales de noticias interesantes sobre cualquiera de las ramas de la Lingüistica Aplicada y de errores de los que se sea testigo durante el cuatrimestre que cometan aprendices de una segunda lengua (hay que intentar analizar esos errores), debates, retos puntuales, etc. (10\% de la nota final)

Para comprobar el estado inicial del conocimiento de la materia por parte del alumnado se le pidió que reflejara lo que supiera inicialmente sobre LA en la primera tarea obligatoria que tenía como fecha de entrega máxima el viernes 16 de octubre. Esa tarea consistió en escribir una autobiografía lingüística en la que también tenía que contestar a preguntas sobre cuestiones clave de la materia conceptuales, procedimentales y actitudinales. También se le pidió a cada estudiante que describiera y evaluara lo que sabía al inicio del cuatrimestre de cada uno de los temas de la LA que aparecen en el programa de la asignatura y en la Figura 1. Se ha pedido al alumnado al final del cuatrimestre que conteste a esas mismas preguntas y que se autoevalúe sobre el conocimiento adquirido en cada una de las ramas de la LA y sobre todo en la que atañe al reto en el que ha trabajado. Desgraciadamente, la fecha límite de entrega de esta publicación establecida por una burocracia que solo atiende a sus intereses y no tiene en cuenta las necesidades y la complejidad de la valoración Ciclos de Mejora en el Aula (2020). Experiencias de Innovación Docente de la US
Esta obra se distribuye con la licencia Creative Commons 
de una innovación docente tan compleja como esta, me ha impedido poder evaluar de forma sosegada lo conseguido en este CIMA ya que el alumnado no ha podido entregar todavía ni su diario reflexivo individual ni su portafolios grupal final, que están en fase de elaboración.

\section{Desafío Transdisciplinar Leln sobre lenguaje inclusivo}

Como se comentó anteriormente, yo me encargué de supervisar el trabajo de los cinco equipos transdisciplinarios que decidieron abordar el desafío marco Leln sobre lenguaje inclusivo. Se puede ver una descripción de este reto-marco en el anexo. De los cinco, solo tres contaban con alumnado de LA.

En el mapa conceptual que sigue a continuación (Figura 2) se puede ver en el centro dos de los retos definidos por dos de esos tres equipos. Uno centrado en el lenguaje inclusivo en el ejército y otro centrado en el lenguaje inclusivo para personas no binarias.

La primera cuestión que aparece en el centro del mapa sobre si se debería reformar la constitución española para reescribirla en un lenguaje más inclusivo es la que dio origen a este desafío interdisciplinar ya que junto con la profesora Ruth Rubio, de Derecho, y Vicente Manzano habíamos organizado para abril de 2020 una mesa de expertos sobre esta cuestión coincidiendo con el informe que la RAE había emitido en febrero sobre esta misma cuestión a petición del gobierno. Este informe, al igual que el de Bosque (2012) fue muy crítico con el lenguaje inclusivo y puso de manifiesto la opinión conservadora de la RAE sobre este tema. El debate se tuvo que posponer al 21 de octubre de 2020 por la pandemia, coincidiendo con el inicio del primer cuatrimestre del curso 2020/2021.

En la parte superior del mapa conceptual de la figura 2 se puede apreciar la relación de este tema con las 
diferentes ramas de la lingüística aplicada. En la parte inferior se describen algunas de las cuestiones fundamentales en las que se centra la polémica. En la derecha, se puede ver la relación con la Hipótesis Sapir-Whorf o de relativismo lingüístico. A la izquierda, se relaciona este desafío marco con el desafío Discursos de Odio, tema sobre el que han trabajado dos de los 28 equipos de la asignatura y 9 de los 37 equipos transdisciplinares con alumnado de otras facultades.

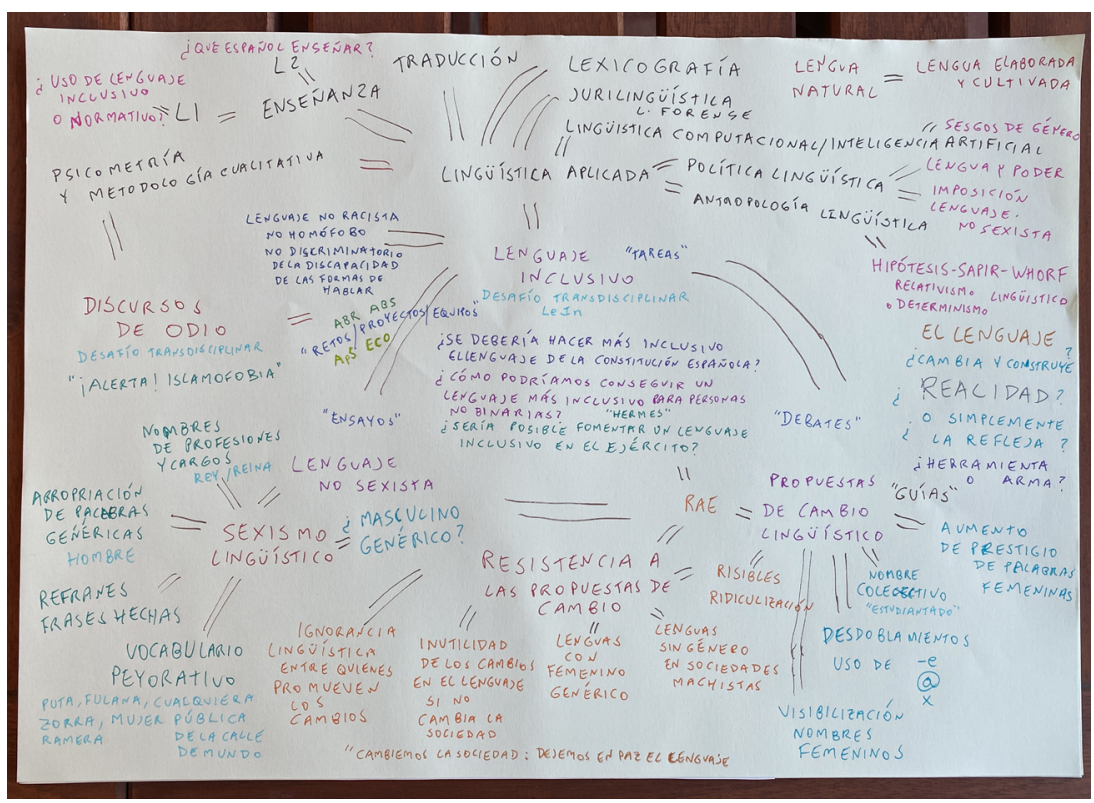

Figura 2 Mapa conceptual sobre lenguaje inclusivo y su relación con la LA

Las secuencia de actividades que se han llevado a cabo para que el alumnado profundice en esta cuestión están señaladas en azul alrededor de las preguntas centrales. Como no puede ser de otra manera, la principal actividad es el diseño y desarrollo por parte de los equipos que así lo han decidido de un reto transdisciplinar para el que se han aliado con alumnado de psicología. No obstante, para que el resto de la clase también aprendiera sobre esta cuestión, se propusieron una serie de tareas, posibles ensayos sobre los que se podía escribir para profundizar Ciclos de Mejora en el Aula (2020). Experiencias de Innovación Docente de la US Reconocimiento-NoComercial-SinObraDerivada Internacional (CC BY-NC-ND 4.0.) 
sobre el tema por parte del alumnado de otros equipos, y se celebró un debate en cada uno de los grupos de la asignatura. Además, se pidió a todo el alumnado que asistiera o al menos viera la grabación del debate del día 21 de octubre y de otro debate entre expertos que se llevó a cabo en la UPO el 17 de diciembre. Tras cada debate, se analizó lo acontecido en clase y se pidió que se comentara comunitariamente también en el Perusall de la asignatura. Por otro lado, en tres de las cuatro sesiones del congreso del 23 de enero el tema del lenguaje inclusivo fue uno de los que más se debatió. Todavía se están recogiendo y analizando las respuestas de las tareas y de las actividades relacionadas con este desafío interdisciplinar con lo que no se podrá hacer un análisis ni una descripción detallada de lo aprendido por el alumnado hasta más adelante.

\section{Evaluación del CIMA}

Todavía es muy precipitado poder evaluar lo conseguido con esta innovación docente ya que el alumnado acaba de terminar la fase OFRECE con el ECOshowUS del 29 de enero de 2021 y por tanto no ha tenido tiempo de reflexionar sobre todo lo vivido y la huella dejada por la asignatura. Por experiencia de cursos anteriores es en la elaboración y entrega del portafolios final grupal cuando el alumnado se da cuenta de todo lo aprendido con lo vivido durante el cuatrimestre. Es por ello que esta sección de evaluación de este CIMA no se podrá completar hasta que no se puedan evaluar adecuadamente los productos finales entregados por los 83 alumnos de la asignatura, los 28 portafolios grupales de cada proyecto/reto, los vídeo-resumen, los pósteres o carteles y los diarios reflexivos individuales que recogerán la huella que ha ido dejando la asignatura. Baste decir por ahora que el ambiente y la motivación que se perciben en la mayoría de los equipos es muy positivo. El docente se ha tenido que emplear a fondo en los problemas que han surgido en el trabajo en equipo con alumnado de otras facultades, pero el esfuerzo ha valido la

Ciclos de Mejora en el Aula (2020). Experiencias de Innovación Docente de la US Esta obra se distribuye con la licencia Creative Commons 
pena. Creemos que la colaboración interdisciplinar y el dar libertad y autonomía para elegir también la metodología han sido un éxito y van a contribuir a un aprendizaje más duradero y significativo por parte del alumnado. Como botón de muestra, sirvan estas palabras de un mensaje recibido por parte de una alumna hace dos días, el viernes 29 de enero: "Quiero darte las gracias una vez más por tu implicación con nuestra educación. Es la asignatura de toda la carrera en la que he aprendido más." La inmensa mayoría de los equipos se han conformado con alumnado de las asignaturas de Psicología y con el alumnado de LA ya que Vicente Manzano y yo fuimos los únicos que obligamos a nuestro alumnado a aventurarse en el trabajo en los equipos transdisciplinares. El alumnado de las asignaturas de Psicometría y Metodología Cualitativa han aportado a mi alumnado de LA el rigor de una metodología basada en el conocimiento de estadística que el alumnado de Filología no recibe. Mi alumnado ha aportado al alumnado de psicología un conocimiento más profundo de los conceptos y temas en los que tenían que basar los constructos que sería necesario medir. Al ser la primera vez que nos hemos embarcado en proyectos transdisciplinarios, no cabe duda que hemos cometido fallos que nos han sido señalados por nuestro alumnado y que intentaremos corregir el curso que viene si tenemos la oportunidad de poder repetir esta enriquecedora experiencia. Las principales y más recurrentes críticas que hemos recibido han tenido que ver con las diferentes expectativas y requisitos establecidos por cada docente en las diversas asignaturas que han participado en esta experiencia y que han llevado a un diferente grado de implicación del alumnado de las diferentes facultades y asignaturas. El curso que viene intentaremos homogeneizar los requisitos y exigencias.

Ciclos de Mejora en el Aula (2020). Experiencias de Innovación Docente de la US Esta obra se distribuye con la licencia Creative Commons 
Palabras clave: lingüística aplicada, docencia universitaria; innovación docente universitaria, lenguaje inclusivo, Aprendizaje Basado en Retos

Keywords: applied linguistics, university teaching, teaching innovation at the university level, inclusive language, Challenge Based Learning

\section{Referencias bibliográficas}

Bain, K. (2007). Lo que hacen los mejores profesores universitarios. Valencia: Universitat de Valencia. Servei de Publicacions.

Bosque, I. (2012). Sexismo lingüístico y visibilidad de la mujer. Boletín de Información Lingüística de la Real Academia Española, nำ1, 1-18.

Herrero-Vázquez, E.-A y Torres-Gordillo, J.-J. (2021). ECO en la educación superior: un aprendizaje resonante. En M. Reyes-Tejedor, D. Cobos-Sanchiz y E. López-Meneses (Coords.), Innovación pedagógica universitaria: reflexiones y estrategias (cap. 8). Editorial Octaedro.

Melero-Aguilar, N., Torres-Gordillo, J.-J. y García-Jiménez, J. (2020). Retos del profesorado universitario en el proceso de enseñanza-aprendizaje: aportaciones del método ECO (Explorar, Crear y Ofrecer). Formación Universitaria, 13(3), 157-168.

Porlán, R. (2017). Enseñanza universitaria. Cómo mejorarla. Madrid: Morata

Torres-Gordillo, J.-J., Melero-Aguilar, N. \& García-Jiménez, J. (2020). Improving the university teaching-learning process with ECO methodology: Teachers' perceptions. Plos One, 15(8), 1-14.

Torres-Gordillo, J.-J., García-Jiménez, J. \& Herrero-Vázquez, E.-A. (2020). Contributions of technology to cooperative work for university innovation with Design Thinking. Pixel-Bit. Revista de Medios y Educación, 59, 27-64.

Torres-Gordillo, J.-J. y Herrero-Vázquez, E.-A. (2021). Innovación metodológica transdisciplinar en la universidad con el método ECO. En Sanchiz y E. López-Meneses (Coords.), Innovación pedagógica universitaria: reflexiones y estrategias (cap. 9). Editorial Octaedro.

Ciclos de Mejora en el Aula (2020). Experiencias de Innovación Docente de la US Esta obra se distribuye con la licencia Creative Commons 


\title{
Anexo
}

\author{
M. Reyes-Tejedor, D. Cobos- \\ Desafíos o Retos Marco interdisciplinares propuestos \\ por el profesorado \\ Desafíos transdisciplinares \\ Código:DobleR \\ Título: $\quad$ Doble realidad
}

\section{Descripción}

Está siendo habitual en la mayoría de países que el inglés se establezca como idioma necesario para satisfacer con éxito facetas fundamentales de la vida. El fenómeno "Doble Realidad" describe la existencia de dos realidades lingüísticas paralelas. Por un lado la cotidianidad se desarrolla exclusivamente en el idioma A, pero el sistema educativo, el mercado laboral y otras instituciones establecen la necesidad de acreditar dominio del idioma B. Esta práctica consiste en la búsqueda de evidencias de la doble realidad en diversos países, junto con la consideración de variables psicosociológicas y políticas. En cada vez más paises existe el requisito legal o práctico de demostrar un nivel suficiente del idioma inglés, basado en su necesidad cotidiana. No obstante, los datos no parecen corroborar esa necesidad, en el sentido de que los idiomas locales parecen suficientes para desempeñarse en la vida cotidiana.

En qué consiste el desafío

Se atraviesan las siguientes fases: (1) elección de al menos dos países donde el inglés no es idioma institucional, y cuyo idioma local (o uno de los locales) es dominado por al menos un miembro del grupo; (2) elección y estudio de materiales editados en el país (periódicos, páginas web, normativa legal...) que versen sobre la necesidad de aprender inglés; (3) elección de al menos dos habitantes de cada país, diversos en edad y género, que posean un trabajo remunerado estable sin relación directa

Ciclos de Mejora en el Aula (2020). Experiencias de Innovación Docente de la US Esta obra se distribuye con la licencia Creative Commons 
con el sector turístico; (4) entrevistas focales para identificar el idioma en la cotidianidad y del trabajo, el papel del inglés en su trayectoria vital, sus opiniones e interpretaciones. El equipo elabora un vídeo de 5 minutos donde se describe el fenómeno de la doble realidad, se describe la experiencia y se exponen los resultados. El vídeo se subtitula en español y en al menos otro idioma dominado por alguien del equipo.

Desafíos transdisciplinares

Código:Leln

Título: Lenguaje inclusivo

\section{Descripción}

Existe un debate abierto muy intenso en torno al concepto de lenguaje inclusivo. Este desafío consiste en conocer el fenómeno, acotar las posiciones y argumentos y estudiar en la práctica en qué medida la población de hablantes sufren esta concreción de discriminación sostenida lingüisticamente. En muchas comunidades lingüísticas se mantiene abierto un debate en torno al lenguaje inclusivo, en la búsqueda de justicia o superación de la discriminación que sufren grupos sociales concretos a través de cómo se ha establecido el uso del idioma materno. El debate llega en muchos casos a una polarización que afecta a la población en general, muchas veces desconocedora de las distintas ideologías que sustentan los usos del lenguaje y el surgimiento de estándares.

En qué consiste el desafío

En acotar bien el fenómeno, coleccionando argumentos a favor y en contra, además de otras posiciones más complejas. Se estudia y describe cómo el lenguaje afecta la realidad o la reproduce, de tal forma que un lenguaje discriminatorio ayuda a mantener una realidad discriminatoria. Se describen las alternativas propuestas y las reacciones que las acompañan. No se trata de un desafío teórico, sino que se acompaña con entrevistas y recogida

Ciclos de Mejora en el Aula (2020). Experiencias de Innovación Docente de la US Esta obra se distribuye con la licencia Creative Commons 
de datos a personas que juegan papeles diferentes. El 21 de octubre tiene lugar una mesa redonda virtual sobre lenguaje inclusivo. El equipo asistirá al acto y recogerá datos, tanto del público como de quienes intervienen en la mesa redonda como ponentes.

Desafios transdisciplinares

Código:DiscursoOdio

Título: $\quad$ Discurso de odio

\section{Descripción}

La situación de inestabilidad política y social nos está llevando hacia la polarización del discurso, lo que ha tenido como consecuencias directas el surgimiento de discursos de odio hacia colectivos que identificamos como contrarios a nosotros, ya sea por razones de género, ideológicas, raciales, etc. Este discurso se está viendo alimentado por el uso de las redes sociales y los medios de masas, destacando el surgimiento de bulos que favorecen el fenómeno de la infoxicación, poniendo a prueba nuestro juicio crítico.

En qué consiste el desafío

El reto será diseñar una campaña de prevención del discurso de odio dirigida a la comunidad universitaria. Para ello, se deberá identificar a un colectivo que sea objeto de discurso de odio y, en base al análisis de los diferentes elementos implicados, conocer las razones profundas de ese odio, detectando los medios por los que se propaga y la intención soterrada de sus instigadores.

Para contribuir a la consecución de este reto, a lo largo del cuatrimestre se anunciarán diferentes seminarios centrados en colectivos que son objeto de discurso de odio, de manera que los alumnos podrán interactuar con especialistas en la materia, estableciéndose así un intercambio de ideas.

Ciclos de Mejora en el Aula (2020). Experiencias de Innovación Docente de la US Esta obra se distribuye con la licencia Creative Commons 


\section{Desafíos transdisciplinares}

\section{Código:Avatar}

Título: Aprender Tu'un Savi, la Lengua de la Lluvia, para sentirnos parte de un pueblo originario que lucha por salvaguardar su territorio y su cultura.

\section{Descripción}

Todo el alumnado conoce la película Avatar (2009) de James Cameron, se ha identificado con la perspectiva del pueblo Na'vi y ha entendido los motivos para defender un planeta amenazado por la destrucción inminente. Hay quienes están pendientes de que aparezca la segunda parte. Pues bien, el mito de Avatar se basa en varias realidades históricas, culturales y lingüísticas: El extractivismo que amenaza la biodiversidad en todo el planeta Tierra y está acelerando el cambio climático: compañías mineras (minas a cielo abierto) y petroleras (campos petrolíferos, arenas bituminosas, oleoductos), eléctricas (grandes presas hidroeléctricas), madereras (papel, mobiliario, construcción, maderas preciosas), ganaderas (talar para sembrar pasto). Las estrategias contemporáneas de los pueblos originarios en Mesoamérica (México, Honduras, Guatemala, etc.) y otras zonas de América del Sur (Ecuador, Chile, el Chaco, Bolivia) o del Norte (USA, Canadá), con el fin de proteger su territorio de las amenazas de destrucción como consecuencia del extractivismo. La vinculación entre la pérdida de biodiversidad y los riesgos que amenazan la diversidad lingüística y cultural, puesto que los planes del extractivismo global implican la expulsión de los pueblos originarios, la apropiación de su territorio, la desintegración comunitaria del pueblo, forzado a migrar y a renunciar a su cultura, así como el despojo de sus medios de subsistencia (en relación menos agresiva con los ecosistemas locales). El valor intrínseco de cada lengua para comprender y atesorar el medio natural, convertido en hábitat de un pueblo, pero también como construcción de un imaginario cultural: una red de espacios mentales, memorias y 
metáforas cognitivas. La película Avatar y el proyecto Ndatiaku nos invitan a que la investigación se ponga al servicio del bien común, de un modo concreto, teniendo en cuenta los eventos del Ñuu Savi ("Pueblo/Comunidad de la lluvia") o Ñuu Ndavi ("Comunidad del pueblo, la gente humilde"). Los paralelos entre los Na'vi y el Ñuu Savi son demasiado extraordinarios para ser casuales. La ventaja del Tu'un Savi, la "lengua de la lluvia": es real.

En qué consiste el desafío

Vamos a aprender paso a paso el Tu'un Savi, no tanto para usarlo en la vida cotidiana, como para comprender la riqueza de una lengua viva en todos sus niveles de construcción:

1. La fonética/fonología de una lengua tonal, que atribuye valor distintivo a la escala tonal en el léxico (los significados) y la sintaxis (por ejemplo, el tiempo y el aspecto verbal), además de en la entonación pragmática. Comprenderemos que los sonidos también son metafóricos: intervienen en la formalización y la comprensión del espacio-tiempo.

2. La semántica cognitiva, que organiza lo que Jerome Bruner llama "Folk Psychology" y la lingüística cognitiva llama "embodiment": el cuerpo individual y colectivo en el medio natural. En consecuencia, cada lengua contiene una visión del mundo insustituible, con sus virtudes atesorables y sus defectos corregibles, como los sesgos machistas. Sin las redes semánticas no serían posibles la empatía y la teoría de la mente.

3. La sintaxis formal que explica la capacidad computacional de nuestra mente para procesar información y la economía creativa de todas las lenguas, desde la perspectiva del minimalismo (la última corriente del generativismo). Haremos comparaciones entre el Tu'un Savi y el español y otras lenguas que conozcamos, para comprobar que existe una $\mathrm{FL}$ (Facultad de Lenguaje con base genética y carácter 
universal en nuestra especie); y para distinguir los parámetros específicos de una u otra lengua.

4. El orden de los argumentos y las redes temáticas de adjetivos y verbos en Tu'un Savi; es decir, los papeles semánticos que seleccionan adjetivos y verbos: agente y paciente, hablante e interlocutor, experimentante y causante, tema de una identificación o un movimiento, locativos, circunstantes temporales, etc. Comprobaremos que sin ellos no es posible ninguna lengua; pero cada lengua los construye de un modo peculiar.

5. La sintaxis temática que organiza la comunicación, por medio de la entonación, con el fin de producir actos de habla e inferencias pragmáticas y hacer posible la progresión temática en un texto. En este terreno queda mucho por investigar.

6. Los géneros discursivos que organizan la sociedad en esferas de comunicación y reparten los roles sociales (el trabajo en la familia, cargos en la comunidad), permiten la iniciación de nuevos miembros (ritos de paso, educación), construyen la identidad colectiva (rituales, etnodramas), hacen posible la transmisión de la memoria cultural y facilitan la adaptación a los cambios económicos, políticos o culturales. Nos sorprenderá conocer cómo los géneros orales se han convertido en géneros digitales vernáculos a través de los medios sociales (Youtube, Facebook, etc.). ¿Y en Instagram o TikTok?

\section{Desafíos transdisciplinares}

Código:Esperanto/ Sistema de Signos Internacional Título: Aprendizaje consciente de algo más que un idioma: Esperanto.

Descripción

Ciclos de Mejora en el Aula (2020). Experiencias de Innovación Docente de la US Esta obra se distribuye con la licencia Creative Commons 
El contenido común es estudiar un idioma desde cero, así como los valores de ciudadanía planetaria que lo acompañan, siendo consciente de los procesos que participan y de cómo la/el estudiante avanza en la adquisición de la lengua y en el contacto con la comunidad hablante, observando los cambios que van ocurriendo a nivel individual y grupal. El esperanto y el sistema de signos internacional son idiomas como cualquier otro, es decir, usados para la cotidianidad, con amplia producción literaria y hablantes nativos. Como particularidades, es hablado por minorías muy activas de los cinco continentes, que tienden a estar en contacto, con abundantes jornadas de todo tipo. Por lo general quienes hablan esperanto/SSI suelen sentirse miembros de la ciudadanía planetaria, tienen un nivel de estudios alto y muchas personas son políglotas. El objetivo manifiesto es proteger la diversidad lingüística del planeta y proponerse como idioma auxiliar para la comunicación internacional e intercultural, pretensión apoyada por la UNESCO. No obstante, un cada vez más nutrido grupo de esperantistas tiende sencillamente a disfrutar del idioma, de su producción cultural y de su comunidad, sin más pretensiones que contar con una red de amistad planetaria. Existe un conjunto muy extenso de recursos para aprenderlo y practicarlo. En España la UNED oferta los niveles A2 y B1. Se acredita siguiendo el Cuadro Europeo de Referencia para la Lenguas, CERL. Si quieres escucharlo a través de música, he aquí unos ejemplos: María Villalón, Martin Wiese, versiones de Aleluya, Hello, o este proyecto de amigos y amigas del mundo e incluso una simpática canción infantil hecha por un par de sevillanos.

En qué consiste el desafío

En aprender el idioma internacional desde cero, procurando un alto nivel de conciencia desde el punto de vista cultural y lingüístico, para remarcar los cambios de aprendizaje que están teniendo lugar. El aprendizaje es gratuito y se llevará a cabo mezclando tres procedimientos: (1) elección de un método autodidacta a elección de

Ciclos de Mejora en el Aula (2020). Experiencias de Innovación Docente de la US Esta obra se distribuye con la licencia Creative Commons 
cada participante; (2) participación en un curso virtual para principiantes; (3) sesiones de práctica en grupos de dos o tres hablantes; (4) cuando se alcance más o menos el nivel $A 1$, conversación virtual con tutores si es posible en SSI.

Desafíos transdisciplinares

Código: Escritura

Título: $\quad$ Escritura masiva

\section{Descripción}

La aparición de la escritura hace más de 8.000 años diferencia la prehistoria de la historia. La humanidad al desarrollar un código de transliteración de la lengua oral a la escrita y registrarlo en un soporte físico (piedra, papiro, electrónico, etc.) permite que ciertos mensajes no desaparezcan a lo largo del tiempo pero no todo son ventajas porque la escritura abre brechas entre a) las lenguas con escritura y las ágrafas (Moreno Cabrera, Juan Carlos 2000: La dignidad e igualdad de las lenguas: critica de la discriminación lingüística, Madrid: Alianza Editorial), las personas alfabetizadas y las analfabetas. Actualmente la escritura ya no tiene solamente un registro académico sino también un registro inmediato. ¿El sistema de la escritura en español responde a las necesidades de ambos tipos de escritura? La capacidad humana para descifrar ese código escrito es la lectura, una capacidad intelectual que implica disponer de tres competencias básicas: una competencia de conocimiento del sistema lingüístico y su adecuada utilización, una competencia cognoscitiva, que supone el conocimiento previo, la experiencia o vivencia que aporta conocimiento y la recuperación de conocimiento memorizado y una competencia comunicativa, que relaciona al texto con el contexto de comunicación como expone Martínez, 1997, p. 108-114 (Disponible en: <http://www.unesco-lectura.univalle.edu.co/articulos.html>

¿En qué consiste el desafío?

Ciclos de Mejora en el Aula (2020). Experiencias de Innovación Docente de la US Esta obra se distribuye con la licencia Creative Commons 
En analizar y describir las brechas que abre la escritura y cómo podemos anularlas o aminorarlas: la escritura como importante fuente de prejuicios lingüísticos que llevan a pensar que las lenguas que carecen de sistema de escritura, son lenguas de poca cultura o incluso lenguas inferiores, que las personas que no poseen o no pueden desarrollar las competencias básicas para alcanzar la escritura son ciudadanos excluidos. Las deficiencias de las reglas ortográficas de la escritura académica para para responder a las necesidades de la escritura inmediata.

Ciclos de Mejora en el Aula (2020). Experiencias de Innovación Docente de la US Esta obra se distribuye con la licencia Creative Commons 